\title{
A dynamic relation between whole-brain white matter microstructural integrity and anxiety symptoms in preadolescent females with pathological anxiety
}

\author{
Nakul Aggarwal $\mathbb{D}^{1 凶}$, Lisa E. Williams ${ }^{1}$, Do P. M. Tromp ${ }^{1}$, Daniel S. Pine ${ }^{2}$ and Ned H. Kalin ${ }^{1}$ \\ (c) The Author(s) 2022
}

Pathological anxiety typically emerges during preadolescence and has been linked to alterations in white matter (WM) pathways. Because myelination is critical for efficient neuronal communication, characterizing associations between WM microstructure and symptoms may provide insights into pathophysiological mechanisms associated with childhood pathological anxiety. This longitudinal study examined 182 girls enrolled between the ages of 9-11 that were treatment-naïve at study entry: healthy controls $(n=49)$, subthreshold-anxiety disorders (AD) $(n=82)$, or meeting DSM-5 criteria for generalized, social, and/or separation ADs ( $n=$ 51), as determined through structured clinical interview. Anxiety severity was assessed with the Clinical Global Impression Scale and Screen for Child Anxiety and Related Emotional Disorders (SCARED). Participants $(n=182)$ underwent clinical, behavioral, and diffusion tensor imaging (DTI) assessments at study entry, and those with pathological anxiety (subthreshold-AD and AD, $n=133$ ) were followed longitudinally for up to 3 additional years. Cross-sectional ANCOVAs (182 scans) examining control, subthreshold-AD, and $A D$ participants found no significant relations between anxiety and DTI measurements. However, in longitudinal analyses of girls with pathological anxiety (343 scans), linear mixed-effects models demonstrated that increases in anxiety symptoms (SCARED scores) were associated with reductions in whole-brain fractional anisotropy, independent of age (Std. $\beta(95 \% \mathrm{Cl})=-0.06(-0.09$ to $-0.03), F(1,46.24)=11.90, P=0.001)$. Using a longitudinal approach, this study identified a dynamic, within-participant relation between whole-brain WM microstructural integrity and anxiety in girls with pathological anxiety. Given the importance of WM microstructure in modulating neural communication, this finding suggests the possibility that WM development could be a viable target in the treatment of anxiety-related psychopathology.

Translational Psychiatry (2022)12:57; https://doi.org/10.1038/s41398-022-01827-y

\section{INTRODUCTION}

Anxiety is dimensional and, when extreme, becomes maladaptive and is pathological. Anxiety disorders (ADs) are among the most common childhood psychiatric illnesses, affecting up to $30 \%$ of youth [1]. In addition, numerous children have subclinical and persistent anxiety symptoms that do not meet DSM-5 criteria [2, 3]. Like children with ADs, these children also suffer considerably and are at increased risk to develop more significant stress-related psychopathology later in life $[2,4,5]$. Because anxiety is dimensional in nature, studying the full range of anxiety may provide insights into the factors that contribute to the varying degrees of distress and disability experienced by children with pathological anxiety. Understanding the factors underlying the development and expression of anxiety in young girls is of particular interest because after the transition to adolescence there is a two-fold increase in the prevalence of ADs in adolescent girls compared to boys that persists throughout the reproductive years [6-8].

White matter (WM) microstructure is highly relevant to adolescent development as adolescence is characterized by changes in WM pathways critical for effective neuronal communication [9-12]. While many studies have examined WM pathways in adults with high trait anxiety and ADs [13-23], considerably less work has examined WM in anxious youth [24-29]. Given evidence linking prefrontal-limbic pathways to anxiety $[21,25,30-36]$, our prior work focused on the uncinate fasciculus (UF), the major WM pathway linking prefrontal regions to temporal lobe structures, including the amygdala and anterior hippocampus [37, 38]. These studies demonstrated anxiety-related reductions in UF fractional anisotropy (FA), a measure of WM microstructural integrity, in adults, preadolescent children, and preadolescent non-human primates (NHPs) [21, 25, 39]. Interestingly, our findings in preadolescent children and preadolescent NHPs suggest that the relation between UF FA and anxiety is present in males but not in females $[25,39]$.

To more comprehensively characterize WM in anxious girls, in the current study we used a longitudinal approach to assess within-participant relations between WM parameters and anxiety symptoms in preadolescent girls (enrolled ages 9-11). Additionally, because of the dimensional nature of anxiety, we included girls with a wide range of anxiety symptoms -

${ }^{1}$ Department of Psychiatry, University of Wisconsin-Madison, 6001 Research Park Boulevard, Madison, WI 53719, USA. ${ }^{2}$ Section on Developmental and Affective Neuroscience, National Institute of Mental Health, Bethesda, MD 20814, USA. ${ }^{凶}$ email: naggarwal5@wisc.edu

Received: 20 August 2021 Revised: 14 January 2022 Accepted: 17 January 2022

Published online: 08 February 2022 
controls (low anxiety), subthreshold-ADs (mild-moderate anxiety), and ADs (meeting DSM-5 AD criteria for generalized, social, and/or separation ADs). We first performed crosssectional analyses comparing WM integrity between girls with ADs, subthreshold-ADs, and controls. Next, in girls with pathological anxiety (subthreshold-AD and AD), using repeated clinical and imaging assessments over a 3-year period, we assessed the longitudinal within-participant relation between anxiety symptoms and WM microstructure. We also assessed relations between WM microstructure with age and pubertal status, which were controlled for in the anxiety-focused analyses.

\section{METHODS}

\section{Participants}

Recruitment and clinical assessment. A total of 182 preadolescent girls with varying levels of anxiety were enrolled between ages 9 and 11 and characterized using clinical, behavioral, developmental, and neuroimaging assessments. Children were recruited from the Madison metropolitan area via community and school advertisements and mass emails. Girls were interviewed with the Kiddie-Schedule for Affective Disorders and Schizophrenia Present and Lifetime version (K-SADS-PL) [40], administered either directly by or under the supervision of a trained PhD-level clinical psychologist or a psychiatrist. Review of audiotapes demonstrated acceptable reliability (Cohen $\mathrm{K}>0.80$ ), and tape review (by DSP) continued throughout the study to maintain interviewer fidelity. Clinicians also rated overall anxiety severity using the Clinical Global Impression Scale-Severity

Table 1. Distribution of $\mathrm{AD}$ Cohort $(n=51)$ by Diagnoses.

\begin{tabular}{|lllllll|}
\hline Total GAD & $\mathbf{1 8}$ & Total SepAD & $\mathbf{2 7}$ & Total SocAD & $\mathbf{2 4}$ & Total Other-Specified AD \\
\hline GAD only & 5 & SepAD only & 14 & SocAD only & 11 & 4 \\
\hline GAD + SepAD & 4 & SepAD + GAD & 4 & SocAD + GAD & 4 & \\
\hline GAD + SocAD & 4 & SepAD + SocAD & 4 & SocAD + SepAD & 4 & \\
\hline GAD + SepAD + SocAD & 5 & SepAD + GAD + SocAD & 5 & SocAD + GAD + SepAD & 5 &
\end{tabular}

$A D$ anxiety disorder, $G A D$ generalized anxiety disorder, SepAD separation anxiety disorder, SocAD social anxiety disorder.

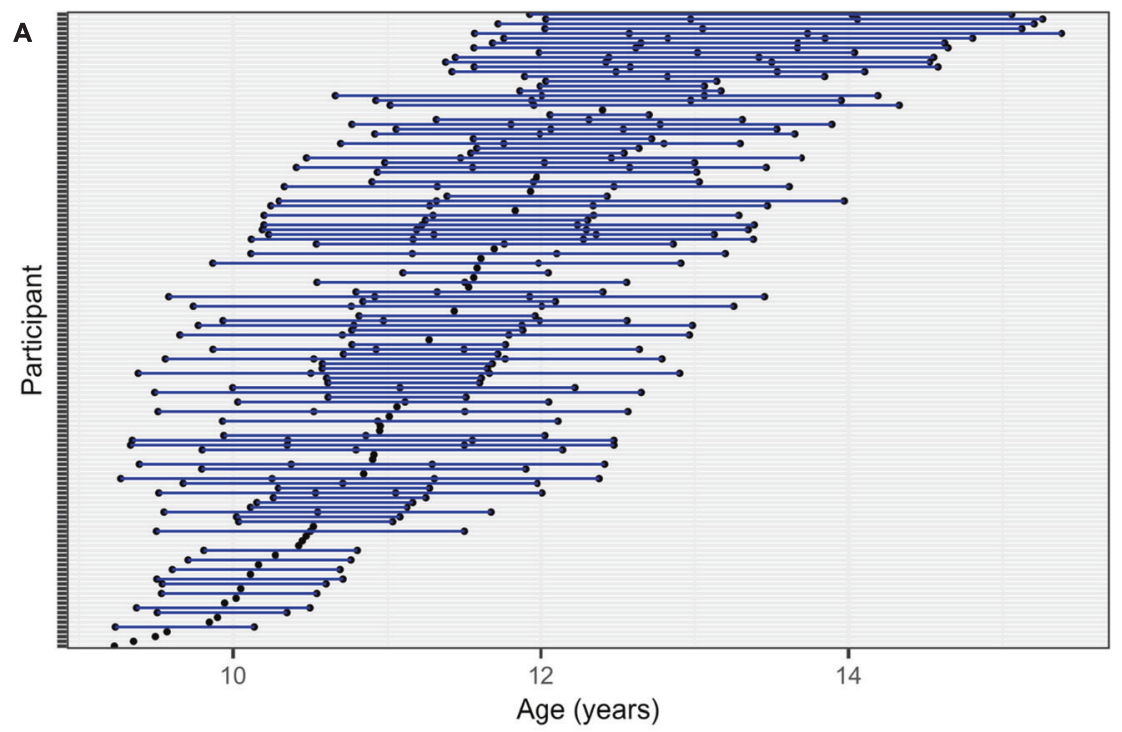

\begin{tabular}{|l|r|}
\hline Measure & Value \\
\hline Participants, No. & 133 \\
\hline Scans per participant, mean (SD) & $2.58(1.18)$ \\
\hline Inter-scan interval, mean (SD), years & $1.16(0.38)$ \\
\hline No. of participants with: & \\
\hline \multicolumn{1}{|l|}{ 1 scan } & 32 \\
\hline 2 scans & 36 \\
\hline 3 scans & 21 \\
\hline 4 scans & 44 \\
\hline Total no. of scans at: & \\
\hline & Time 1 \\
\hline Time 2 & 131 \\
\hline Time 3 & 95 \\
\hline Time 4 & 64 \\
\hline
\end{tabular}

Fig. 1 Depiction of the longitudinal study design. A Each blue line represents one participant; each point represents a scan for the respective participant and indicates age at scan. B Table with descriptive longitudinal scanning information. 
(CGI-S) [41]. Diagnoses and CGI-S ratings were reviewed and confirmed in group discussions with all study clinicians. Included participants had never received treatment for anxiety or other psychiatric illness, were not treatment seeking, and were eligible for an MRI scan. Major exclusion criteria included psychotropic medication use, severe psychopathology in need of immediate treatment, and diagnoses of major depressive disorder, obsessive compulsive disorder, post-traumatic stress disorder, autism, bipolar disorder, or schizophrenia. Informed assent and consent were obtained from all participants and their parents, in accordance with the Institutional Review Board of the University of Wisconsin-Madison. Individuals were compensated for their time and effort.

Based on the K-SADS and CGI-S, participants were categorized into three groups: (1) healthy controls, (2) subthreshold-AD, and (3) AD. Healthy control participants exhibited very minimal, if any, symptoms of anxiety or any other psychiatric illness (CGI-S $=1$, normal/not at all ill). Subthreshold-AD participants exhibited subsyndromal but persistent levels of symptoms associated with generalized, separation, and/or social anxiety but did not meet DSM-5 criteria for these disorders ( $\mathrm{CGI}-\mathrm{S}=2$, borderline mentally ill; or $\mathrm{CGI}-\mathrm{S}=3$, mildly ill). A small number of girls in the subthreshold $\mathrm{AD}$ group $(n=5)$ met criteria for specific phobia. AD participants met full DSM- 5 criteria for generalized anxiety disorder, separation anxiety disorder, and/or social anxiety disorder (CGI-S $>4$, moderately ill or worse). A full description of the number of participants with each $A D$ diagnosis or combination of $A D$ diagnoses can be found in Table 1. Participants in the AD group could have comorbid diagnoses of attention deficit hyperactivity disorder and oppositional defiant disorder if symptoms were less severe than the AD. By design, girls with subthreshold-ADs did not meet full DSM-5 criteria for any disorders at study entry other than specific phobia. However, during longitudinal follow-up, 17 of the 82 girls $(21 \%)$ in the subthreshold-AD group developed ADs or other psychiatric disorders. The final sample $(n=182)$ was comprised of 49 controls, 82 subthreshold-AD, and 51 AD girls who completed the initial year of the study. Girls with pathological anxiety (subthreshold-AD and $A D, n=133$ ) were followed longitudinally for up to 3 years with annual clinical, neuroimaging, and behavioral assessments, including the K-SADS/CGI and multimodal imaging session. 131 participants were assessed in year 1, 95 in year 2,64 in year 3, and 53 in year 4, for a total of 343 scans in the longitudinal sample (Fig. 1). There were no significant differences in anxiety severity at study entry (child SCARED) among children who completed the full study (all 4 scans) vs. those who completed either 1, 2, or 3 scans (Supplementary Fig. 1).

Rating scales. Children's anxiety symptoms were rated by both the child and a parent using the Screen for Child Anxiety and Related Emotional Disorders (SCARED) [42]. Depressive symptoms, environmental stressors, and externalizing behaviors were also assessed using the Child Depression Inventory (CDI)
[43], Stressful Life Events Schedule (SLES) [44], and Conners' Parent Rating Scale-Revised (CPRS-R) [45], respectively. The average interval between SCARED survey completion and MRI scan was 8.83 days (range: $0-178$ days). Children's pubertal status was measured with the Tanner Staging Scale and the Pubertal Development Scale (PDS), completed by child and parent together [46]. These data were collected and managed using REDCap (Research Electronic Data Capture) tools hosted at the University of Wisconsin-Madison, School of Medicine and Public Health, a secure, web-based application designed to support data capture for research studies $[47,48]$.

\section{MRI data acquisition and processing}

DTI acquisition. All brain images were collected on a 3.0 Tesla GE MR750 scanner (GE Healthcare; Waukesha, WI) using a 32-channel head coil. Diffusion-weighted MRI scans were obtained using a two-dimensional echo planar imaging diffusion-weighted spin-echo sequence with 48 optimal non-collinear directions (see Supplementary Methods).

DTI processing and analysis. Image processing was completed using procedures described previously in Tromp et al., 2019 [25], with an additional step of within-participant co-registration of tensor images prior to coregistration across participants. Deterministic tractography was performed in TrackVis [49] to delineate whole-brain WM and seven bilateral tracts of interest across the brain. The 7 WM tracts were selected based on previous literature implicating alterations in these tracts in $\mathrm{ADs}$ and other internalizing disorders, including the UF $[21,25,39]$, cingulum bundle (CING) $[14,26]$, superior longitudinal fasciculus (SLF) [13, 29], stria terminalis/fornix (STRIA/FX) [15], inferior fronto-occipital fasciculus (IFO) [29, 50], internal capsule (IC) $[29,51]$, and corpus callosum (CC) $[17,26]$. For each image, a weighted mean was calculated per tract for each diffusion metric (fractional anisotropy [FA], mean diffusivity $[M D]$, radial diffusivity [RD]) (see Supplementary Methods).

To identify potential anxiety-related WM alterations in regions outside the 7 pre-determined WM tracts, whole-brain voxel-wise FA analyses were performed both cross-sectionally (with all data collected at study entry) and longitudinally (with data from subthreshold-AD and $A D$ participants), using the randomize program in FSL [52] and the 3dLMEr program in AFNI [53], respectively. Input data for both analyses consisted of normalized FA images in standard MNI space (i.e., 182 and 343 FA images included in the crosssectional and longitudinal analyses, respectively).

\section{Statistical analysis}

Cross-sectional analyses ( $n=182$ scans) assessed group differences in WM microstructure and behavioral metrics in the full initial sample of participants (controls vs. subthreshold-AD vs. AD). One-way ANCOVA models assessed

Table 2. Sample characteristics by cohort at study entry.

\begin{tabular}{|c|c|c|c|c|}
\hline Clinical Measure & Healthy Control $(n=49)$ & Subthreshold-AD ( $n=82$ ) & $A D(n=51)$ & $\begin{array}{l}\text { One-Way ANOVA } \\
P \text { value }\end{array}$ \\
\hline IQ (WASI), mean (SD) & $113.92(12.58)$ & $116.77(16.36)$ & $113.94(14.11)$ & 0.449 \\
\hline Tanner Staging scores, mean (SD) & $1.64(0.76)$ & $1.80(0.74)$ & $1.82(0.93)$ & 0.446 \\
\hline Parent SCARED (Anxiety), mean (SD) & $3.04(2.98)$ & $18.93(9.17)$ & $31.02(10.50)$ & $<0.001^{\mathrm{b}}$ \\
\hline CDI (Depression), mean (SD) & $40.40(3.85)$ & $44.74(5.71)$ & $50.02(8.86)$ & $<0.001^{b}$ \\
\hline $\begin{array}{l}\text { ACE-Related SLES (Life Stressors) Count, } \\
\text { mean (SD) }\end{array}$ & $1.57(1.89)$ & $3.10(2.85)$ & $3.63(3.57)$ & $0.001^{c}$ \\
\hline CPRS (ADHD), mean (SD) & $45.40(3.75)$ & $55.25(9.98)$ & $58.90(11.98)$ & $<0.001^{d}$ \\
\hline \multicolumn{5}{|c|}{$\begin{array}{l}\text { WASI Wechsler abbreviated scale of intelligence, } P D S \text { pubertal development scale, SCARED screen for child anxiety related emotional disorders, } C G I-S \text { clinica } \\
\text { global impression scale-severity, } C D I \text { child depression inventory, ACE adverse childhood experiences, } S L E S \text { stressful life events schedule, } C P R S-R \text { Conners' parent } \\
\text { rating scale-revised. } \\
\text { a'Significant main effect of group }(P<0.05) \text { in one-way ANOVA. Post-hoc Tukey indicates subthreshold-AD }>\text { controls }(P=0.007) \text {. } \\
\left.{ }^{\mathrm{b}} \text { Significant main effect of group }(P<0.001) \text { in one-way ANOVA. Post-hoc Tukey indicates stepwise progression (controls }<\text { subthreshold-AD }<\text { AD; all } P<0.001\right) \\
\text { 'Significant main effect of group }(P=0.001) \text { in one-way ANOVA. Post-hoc Tukey indicates subthreshold-AD and AD groups do not differ, but both are higher } \\
\text { than controls }(P<0.05) \text {. } \\
{ }^{\mathrm{d}} \text { Significant main effect of group }(P<0.001) \text { in one-way ANOVA. Post-hoc Tukey indicates subthreshold-AD and AD groups do not differ, but both are higher } \\
\text { than controls }(P<0.001) \text {. }\end{array}$} \\
\hline
\end{tabular}


Table 3. Group differences in tract FA and dimensional relations with SCARED scores at study entry ${ }^{a}$.

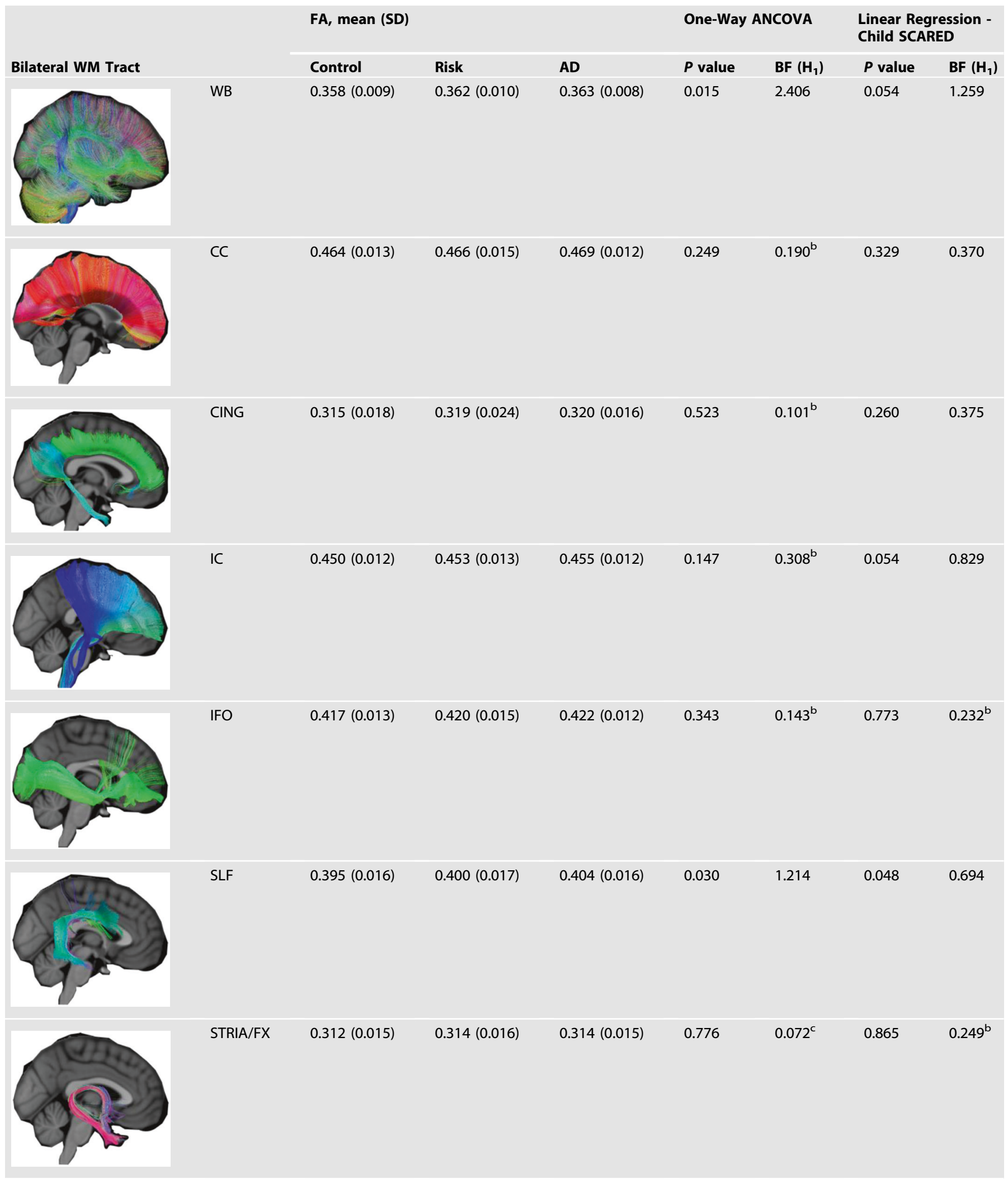


Table 3. continued

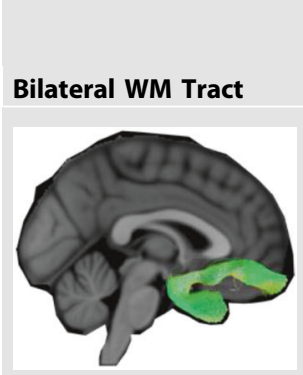

FA, mean (SD)

One-Way ANCOVA

Linear Regression Child SCARED

\section{Control}

Risk

AD

$0.359(0.014)$
$P$ value

0.186
BF $\left(\mathbf{H}_{1}\right)$

$0.254^{\mathrm{b}}$
$P$ value

0.930
BF $\left(\mathrm{H}_{\mathbf{1}}\right)$

$0.230^{\mathrm{b}}$

CC corpus callosum, CING cingulum, IC internal capsule, IFO inferior fronto-occipital fasciculus, SLF superior longitudinal fasciculus, STRIA/FX stria terminalis/ fornix, UF uncinate fasciculus, WB whole-brain WM.

${ }^{\mathrm{a}}$ All analyses control for age at scan. 3-D renderings (right sagittal views) generated from deterministic tractography are shown for each WM tract of interest and whole-brain WM. Green fibers extend along anterior-posterior axis; red fibers along the medial-lateral axis; and blue fibers along the superior-inferior axis.

${ }^{b}$ Moderate evidence of absence of an effect under the Bayesian framework $\left(0.1<\mathrm{BF}_{\mathrm{H}_{1}}<0.33\right)$.

'Strong evidence of absence of an effect under the Bayesian framework $\left(\mathrm{BF}_{\mathrm{H} 1}<0.1\right)$.

between-group differences in: (1) demographic and clinical variables and (2) DTI metrics in the 7 WM tracts and whole-brain WM. Because child- and parent-rated SCARED scores were significantly correlated (see Supplementary Results), and because there is some evidence to suggest that child self-reports may be particularly relevant during this developmental period [54-56], childrated SCARED scores were selected as the primary metric of anxiety severity. Analyses were also performed with parent-rated SCARED scores and are reported below. Linear regression models assessed the between-participant relationship between anxiety levels (child SCARED) and DTI metrics. Because age, PDS scores, and Tanner Staging scores were significantly inter-correlated (see Supplementary Results), age was used as the developmental covariate for all ANCOVA and regression models. Similar analyses substituting PDS or Tanner scores in place of age did not change the results (Supplementary Fig. 2). All models were evaluated using both frequentist and Bayesian statistics, the latter of which affords the ability to claim evidence of absence of an effect (see Supplementary Methods) [57]. Analyses were conducted using the stats and base packages in RStudio (ver. 1.4.1106) and JASP software (ver. 0.14.1). In the cross-sectional voxel-wise analysis, general linear models (using permutation methods [58]) were implemented with the FSL randomise tool [52] to estimate the relationship between child SCARED scores while controlling for age. Using threshold-free cluster enhancement (TFCE) [59], results were assessed at a family-wise error (FWE)-corrected threshold of $P<0.05$.

Across the sample of girls with pathological anxiety (subthreshold-AD and AD participants; $n=343$ scans), longitudinal within-participant relationships between age, child SCARED scores, and WM microstructure in the 7 WM tracts of interest and whole-brain were assessed using linear mixed-effects (LME) models, which allow for precise and unbiased effect estimates by accounting for repeated within-participant measures [60]. As in the cross-sectional analysis, within the longitudinal data age, PDS scores, and Tanner Staging scores were highly inter-correlated (see Supplementary Results), and age was used as the developmental covariate. Separate LME models quantified the withinparticipant relationship between: (1) age and child SCARED scores; and (2) child SCARED scores and WM microstructure in each tract, while controlling for age (see Supplementary Methods). LME modeling was performed using the Ime4 and car packages in RStudio (ver. 1.4.1106) with frequentist statistics. Tractography-based DTI analyses used a Bonferroni-adjusted $P$ value for multiple comparison correction (8 comparisons across 7 tracts and whole-brain WM; $P_{\text {corrected }}<0.05 / 8=0.00625$ ). Within the longitudinal sample of girls with pathological anxiety, LME modeling was also performed at the whole-brain voxel-wise level with the 3dLMEr package in AFNI [53], estimating the withinparticipant relationship between child SCARED scores and FA in each voxel while controlling for age. Results were assessed at both the FDR-corrected level and at an uncorrected threshold of $P<0.005$.

\section{RESULTS}

Cross-sectional analysis (controls vs. subthreshold-AD vs. AD) Groups did not differ in any demographic variable, except PDS scores (subthreshold-AD > control) (Table 2). As expected, groups differed on multiple clinical measures, in most cases in a stepwise manner (AD >
sub-threshold-AD $>$ control) (Table 2). For categorical (control vs. subthreshold-AD vs. AD) or dimensional (child SCARED) analyses, there were no statistically significant associations between anxiety and FA in the WM tracts of interest or in whole-brain WM after multiple comparison correction (Table 3). Bayesian analyses support the interpretation of these null effects as the absence of a relation between anxiety and FA in the CC, CING, IC, IFO, STRIA/FX, and UF $\left(\mathrm{BFs}_{\mathrm{H} 1}<0.33\right)$, and a lack of evidence for anxiety-FA relations in the SLF and whole-brain WM $\left(0.33<\mathrm{BFs}_{\mathrm{H} 1}<3\right)$. As expected, age was associated with FA at the whole-brain level, as well as in the CING, IC, IFO, and UF, but not in the CC, SLF, or STRIA/FX (Supplementary Fig. 3 and Supplementary Table 1). Additionally, voxel-wise analyses did not show any significant FWE-corrected clusters in which child SCARED scores predicted FA.

\section{Longitudinal analysis in girls with pathological anxiety}

In our longitudinal sample of girls with subthreshold-ADs or ADs ( $n=133)$, we examined the within-participant relations between changes in the severity of anxiety symptoms and associated WM microstructural changes throughout the brain while controlling for age. Child SCARED scores exhibited a significant negative correlation with whole-brain FA at the individual level (Std. $\beta$ $(95 \% \mathrm{Cl})=-0.06(-0.09$ to -0.03$), F(1,46.24)=11.90, P=0.001)$, such that increases in a child's anxiety level predicted decreases in her whole-brain FA (Table 4 and Fig. 2). While not statistically significant after multiple comparison correction, parallel analyses with $\mathrm{MD}$ and RD demonstrated consistent results (Supplementary Fig. 4 and Supplementary Table 2). Parallel analyses in each of the 7 WM tracts of interest did not reveal any statistically significant anxiety-FA associations after multiple comparison correction (Table 4). However, at the uncorrected level, multiple WM tracts - including the CC, CING, IFO, and SLF - showed reductions in FA in relation to anxiety severity (Table 4). Substituting parent SCARED scores for child SCARED scores in this analysis also revealed a negative within-participant association between anxiety and whole-brain FA but did not reach statistical significance $(P=0.18)$ (Supplementary Fig. 5). To complement these tract-based analyses, we also performed a voxel-wise analysis across the entire brain. Voxel-wise analyses did not reveal any significant FDR-corrected clusters in which child SCARED scores predicted FA. At an uncorrected threshold $(P<0.005)$, a number of small clusters ( $<120$ voxels) distributed across the brain were negatively associated with child SCARED scores.

While all participants were treatment-naïve at study entry, a subset $(n=27)$ began receiving behavioral and/or pharmacological therapy in follow-up years of the study. In a supplemental 
Table 4. Average within-participant associations between SCARED scores and tract FA ${ }^{a}$.

Bilateral WM Tract
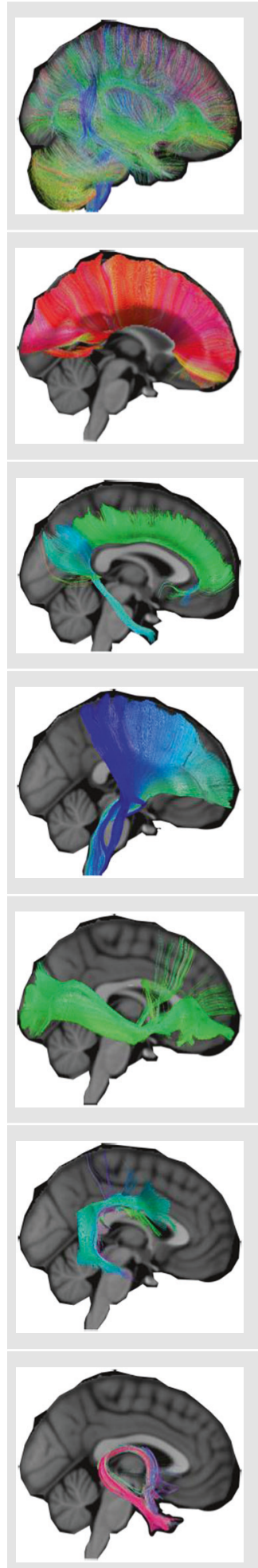

\section{B1}

WB

$-8.438 \mathrm{E}-05$

$-1.057 \mathrm{E}-04$

CC

CING

$-1.027 \mathrm{E}-04$

$-0.03(-0.06$ to -0.01$)$

$-0.02(-0.06$ to 0.01$)$

0.220

IC

IFO

SLF

$-0.03(-0.07$ to 0.00$)$

0.044
$-7.819 \mathrm{E}-05$

$-1.072 \mathrm{E}-04$

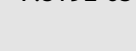

\section{Std. $\beta 1$ (95\% Cl)}

-0.06 ( -0.09 to -0.03$)$

$P$ value $0.001^{\mathrm{b}}$ 
Table 4. continued

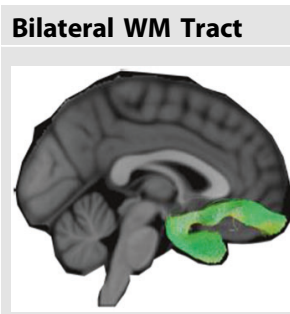

及1

$-4.753 \mathrm{E}-05$
Std. $\beta 1(95 \% \mathrm{Cl})$

$-0.02(-0.06$ to 0.02$)$
$P$ value

0.345

CC corpus callosum, CING cingulum, IC internal capsule, IFO inferior fronto-occipital fasciculus, SLF superior longitudinal fasciculus, STRIA/FX stria terminalis/ fornix, UF uncinate fasciculus, WB whole-brain WM.

${ }^{a}$ All analyses control for age at scan. 3-D renderings (right sagittal views) generated from deterministic tractography are shown for each WM tract of interest and whole-brain WM. Green fibers extend along anterior-posterior axis; red fibers along the medial-lateral axis; and blue fibers along the superior-inferior axis.

${ }^{\mathrm{b}}$ Statistically significant under the frequentist framework at a Bonferroni-corrected level $(P<0.00625)$.

analysis, excluding scans collected after treatment initiation did not alter the findings (Supplementary Fig. 6). Supplementary analyses that included depression and stressful life events as within-participant covariates in separate models did not change the overall pattern of results (see Supplementary Results). While there was a positive within-participant relationship between age and whole-brain FA (Std. $\beta(95 \% \mathrm{Cl})=0.08(0.05$ to 0.12$), F(1$, $64.90)=24.75, P<0.001)-$ in line with the cross-sectional analysis - there was no significant correlation between age and child SCARED scores (Supplementary Fig. 7 and Supplementary Table 3).

\section{DISCUSSION}

This study in young females is one of the largest longitudinal neuroimaging studies of pathological anxiety, focused on understanding alterations in neural pathways relevant to the development of anxiety during childhood. The major finding from this study was derived from the longitudinal data, revealing that within participants, more severe anxiety symptoms were associated with lower whole-brain FA (Table 4 and Fig. 2). This relation manifested in treatment-naïve girls, including girls with subthreshold $A D$ symptoms as well as girls who met criteria for ADs, independent of age and pubertal status. We also performed crosssectional analyses with data collected at study entry, comparing WM integrity among controls, subthreshold-AD participants, and AD participants. In contrast to the longitudinal finding, this crosssectional analysis revealed no significant relations between FA and anxiety. Taken together, these findings highlight a dynamic relation between whole-brain WM integrity and anxiety, as well as the importance of a longitudinal within-participant approach for studying developmental psychopathology.

The presence of a significant longitudinal association between anxiety and WM in the context of no significant cross-sectional association is notable (Tables 1,3 ). This suggests that within an individual, whole-brain FA and anxiety fluctuate together over time, regardless of individual differences in FA magnitude. WM microstructural integrity is influenced by genetic, experiential, and environmental factors. Given the sensitivity of WM to these variables, it is possible that between-subject variation in FA, which is typically greater than within-subject variation, could be due to differences in the extent to which children are impacted by factors that influence myelin development. This could account for our lack of a betweenparticipant finding in the presence of a within-participant, longitudinal finding. We are unaware of other studies that have concomitantly examined the relation between anxiety symptoms and WM parameters longitudinally in children with pathological anxiety. One longitudinal DTI study in a normative sample of youth (ages 6-18) found that children with higher anxiety/depression symptoms at study intake had slower rates of WM development in multiple WM tracts [27]. Another study in a large sample of youth reported an association between initially assessed internalizing and externalizing symptoms with reduced growth-related increases in global WM [61]. Other work examining WM alterations in relation to pediatric anxiety has been cross-sectional. Four cross-sectional DTI studies have been performed in youth (ages 6-18), examining typically developing youth with trait anxiety as well as children with ADs $[25,26,28,29]$. Consistent with DTI studies of anxiety in adults $[13,16,17,19]$, these studies have generally reported anxiety-related WM reductions in FA in various regions, including the UF, CING, CC, and IFO. We previously reported a reduction in UF FA in boys with ADs but not girls, also using a cross-sectional approach [25]. The lack of a relation between anxiety and UF FA in girls in our previous study is consistent with the cross-sectional results reported here in preadolescent girls with pathological anxiety. These null effects should be interpreted cautiously. We note that a Bayesian analysis performed on the data from the girls in the previous study did not support evidence for the absence of an effect (see Supplementary Results), whereas a Bayesian analysis performed on the current dataset was supportive of evidence of absence for the lack of an association between UF FA and anxiety (Table 3).

The correlation between global WM microstructure and anxiety symptoms suggests the presence of a diffuse whole-brain WM effect. Such an effect could have consequences for the inter- and intra-connectivity among brain networks relevant to emotional information processing and integration, aversive stimulus detection, and the interpretation of social behavior. Indeed, similar whole-brain WM microstructural alterations have been reported in relation to general psychopathology factors and cognitive abilities in youth $[24,62]$. The tractography-based findings and whole-brain voxel-wise analysis are consistent with a distributed pattern of alterations in structural connectivity related to changes in anxiety on an individual level. This finding in girls with pathological anxiety contrasts with our previous report of UF-specific reductions in WM integrity in anxious boys, which implicates PFC-limbic alterations in pathological anxiety. The absence of this structural finding in girls does not preclude the possibility that functional studies might reveal other PFC-limbic alterations.

While our results suggest a dynamic relationship between WM microstructure and childhood anxiety, the mechanism underlying this association is unclear. Furthermore, it is possible that the association between WM integrity and anxiety symptoms is not causally linked, as other factors could concomitantly impact both of these measures. However, studies in both NHPs and humans suggest that stress can affect WM microstructure $[63,64]$. As such, it is plausible that in our sample of girls, the experience of chronically heightened anxiety could result in altered WM microstructure. Specific mechanisms that have been implicated from preclinical studies link adversity to WM microstructure via 
A
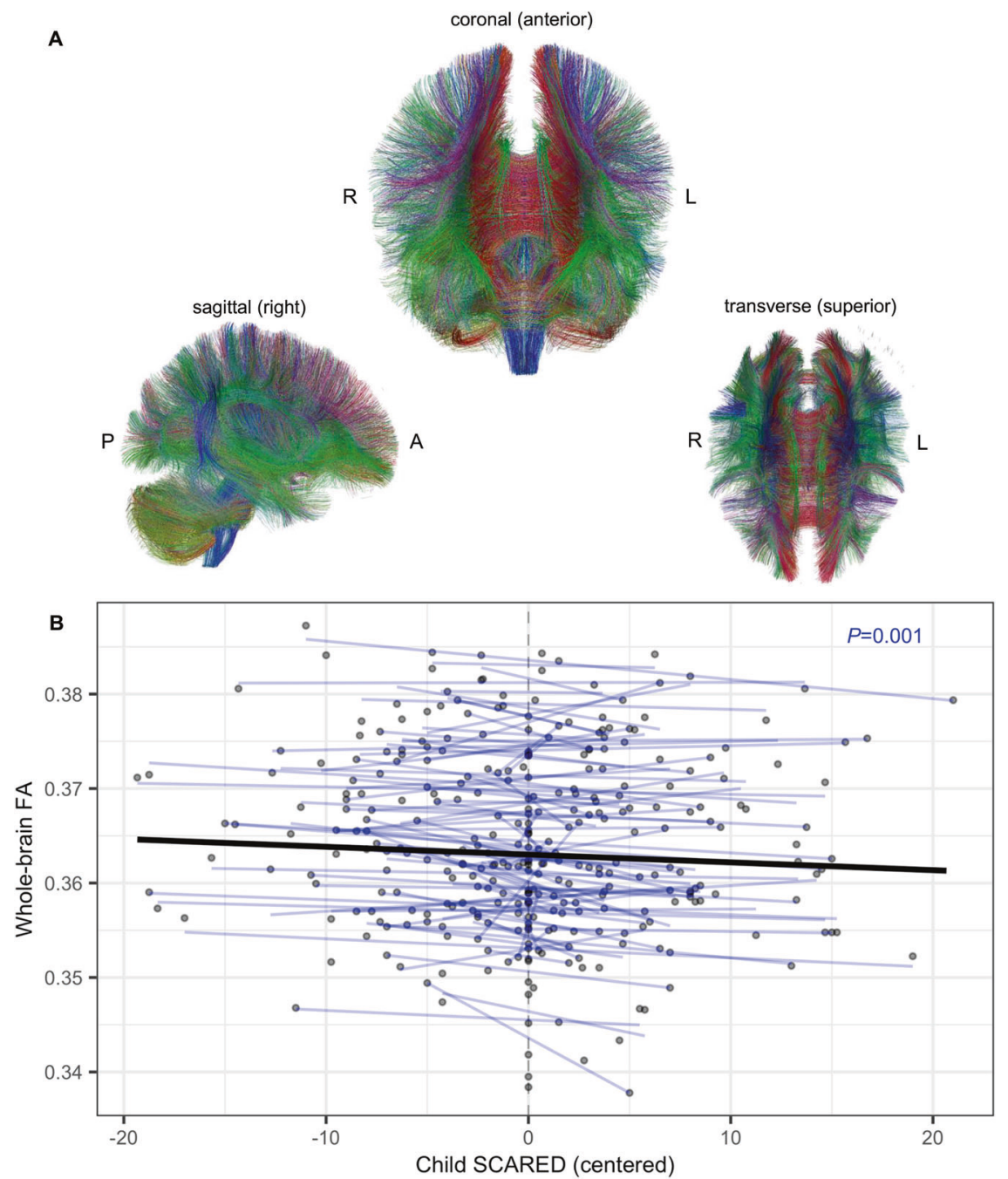

Fig. 2 Longitudinal within-participant association of whole-brain FA with child SCARED scores. A Coronal, sagittal, and transverse views of a 3-D rendering of whole-brain WM tracts, as generated via deterministic tractography in TrackVis (A-P anterior-posterior; R-L right-left). Green fibers extend along anterior-posterior axis; red fibers along the medial-lateral axis; and blue fibers along the superior-inferior axis. B Withinparticipant relationship between whole-brain FA and child SCARED scores. Each blue line represents a participant-specific regression line predicting whole-brain FA from within-participant centered child SCARED scores, while controlling for age. Each point represents an individual scan. The bolded black line depicts the average within-participant association of whole-brain FA with child SCARED scores.

effects on oligodendrogenesis and myelination $[65,66]$. It is also conceivable that WM microstructure plays a role in directly mediating levels of anxiety. Studies manipulating oligodendrocyte function in preclinical models of anxiety could be informative in this regard. Our results provide an impetus to examine the potential utility of broadly targeting WM microstructure in the treatment for early-life anxiety. In this vein, recent work demonstrates that WM is remarkably plastic - particularly in youth - and responsive to various types of cognitive and motor training [67-70]. These studies lay the foundation for future research in clinical samples examining the extent to which existing cognitive and pharmacological therapies for anxiety may exert their effects in part by modulating WM microstructure. Studies in animal models suggest that stressors can impair myelination that is associated with reductions in social behavior $[65,71,72]$. Furthermore, pharmacological agents, including muscarinic antagonists such as clemastine and solifenacin, have been demonstrated to promote oligodendrocyte differentiation and enhance myelination [71, 73-75], and these effects are linked to the recovery of behavioral alterations [71, 73]. This pharmacological strategy could be used in conjunction with specific psychotherapeutic and/or training interventions that are targeted at maladaptive anxiety to potentially augment the reported effects of training and experience on WM integrity in pathways involved in mediating anxiety.

Although this study included a relatively large sample, most participants were White (Supplementary Table 4). Expanding the diversity of the participants in these studies to include more BIPOC individuals will be important to enhance generalizability of the findings. Our sample did not include boys, and our longitudinal analysis did not include control participants, limiting our conclusions regarding the association between WM microstructural integrity and anxiety symptoms to only girls with pathological anxiety. While there was some attrition in the follow-up years of the study, the statistical approaches used - linear mixed-effects models in particular - are designed to handle missing data in longitudinal datasets [60]. While we attribute the FA-anxiety relation to whole-brain reductions in $\mathrm{FA}$, it is possible that there are associations between anxiety symptoms and FA in specific WM tracts that were not examined in this study. 
In summary, we present one of the largest longitudinal neuroimaging studies of pediatric anxiety, demonstrating that, on an individual level in girls with pathological anxiety, worsening of anxiety symptoms is associated with a global decrease in WM microstructural integrity. Importantly, this relationship is independent of age and puberty. An extensive body of literature has shown that childhood and early adolescence are periods of significant WM growth across the brain $[12,76,77]$. Our present results demonstrate that within this overarching developmental pattern, individual variations in whole-brain WM are dynamically linked to childhood anxiety symptom severity. These findings support future studies investigating the possibility of targeting WM as a modality to aid in the prevention and treatment of childhood anxiety disorders.

\section{CODE AVAILABILITY}

Image processing and statistical code used for these studies, as well as imaging data, may be able to be shared with interested parties upon request by contacting the corresponding author.

\section{REFERENCES}

1. Kessler RC, Petukhova M, Sampson NA, Zaslavsky AM, Wittchen H-U. Twelvemonth and lifetime prevalence and lifetime morbid risk of anxiety and mood disorders in the United States. Int J Methods Psychiatr Res. 2012;21:169-84.

2. Haller H, Cramer H, Lauche R, Gass F, Dobos GJ. The prevalence and burden of subthreshold generalized anxiety disorder: a systematic review. Bmc Psychiatry. 2014;14:128.

3. Angst J, Merikangas KR, Preisig M. Subthreshold syndromes of depression and anxiety in the community. J Clin Psychiatry. 1997;58:6-10.

4. Kessler RC, Avenevoli S, Green J, Gruber MJ, Guyer M, He Y, et al. National comorbidity survey replication adolescent supplement (NCS-A): III. Concordance of DSM-IV/CIDI diagnoses with clinical reassessments. J Am Acad Child Adolesc Psychiatry. 2009;48:386-99.

5. Stein MB, Stein DJ. Social anxiety disorder. Lancet. 2008;371:1115-25.

6. Deardorff J, Hayward C, Wilson KA, Bryson S, Hammer LD, Agras S. Puberty and gender interact to predict social anxiety symptoms in early adolescence. J Adolesc Health. 2007;41:102-4.

7. Reardon LE, Leen-Feldner EW, Hayward C. A critical review of the empirical literature on the relation between anxiety and puberty. Clin Psychol Rev. 2009;29:1-23.

8. Copeland WE, Angold A, Shanahan L, Costello EJ. Longitudinal patterns of anxiety from childhood to adulthood: the great smoky mountains study. J Am Acad Child Adolesc Psychiatry. 2014;53:21-33.

9. Herting MM, Maxwell EC, Irvine C, Nagel BJ. The impact of sex, puberty, and hormones on white matter microstructure in adolescents. Cereb Cortex. 2012;22:1979-92.

10. Giorgio A, Watkins KE, Chadwick M, James S, Winmill L, Douaud G, et al. Longitudinal changes in grey and white matter during adolescence. Neuroimage. 2010;49:94-103.

11. Oyefiade AA, Ameis S, Lerch JP, Rockel C, Szulc KU, Scantlebury N, et al. Development of short-range white matter in healthy children and adolescents: shortrange white matter in children and adolescents. Hum Brain Mapp. 2017;39:204-17.

12. Lebel $C$, Beaulieu C. Longitudinal development of human brain wiring continues from childhood into adulthood. J Neurosci. 2011;31:10937-47.

13. Baur V, Hänggi J, Rufer M, Delsignore A, Jäncke L, Herwig U, et al. White matter alterations in social anxiety disorder. J Psychiatr Res. 2011;45:1366-72.

14. Zhang Y, Li L, Yu R, Liu J, Tang J, Tan L, et al. White matter integrity alterations in first episode, treatment-naive generalized anxiety disorder. J Affect Disord. 2013;148:196-201.

15. Modi S, Trivedi R, Singh K, Kumar P, Rathore RKS, Tripathi RP, et al. Individual differences in trait anxiety are associated with white matter tract integrity in fornix and uncinate fasciculus: preliminary evidence from a DTI based tractography study. Behav Brain Res. 2013;238:188-92.

16. Baur V, Brühl $A B$, Herwig $U$, Eberle $T$, Rufer $M$, Delsignore $A$, et al. Evidence of frontotemporal structural hypoconnectivity in social anxiety disorder: a quantitative fiber tractography study. Hum Brain Mapp. 2013;34:437-46.

17. Westlye LT, Bjørnebekk A, Grydeland H, Fjell AM, Walhovd KB. Linking an anxietyrelated personality trait to brain white matter microstructure: diffusion tensor imaging and harm avoidance. Arch Gen Psychiat. 2011;68:369-77.
18. Lu M, Yang C, Chu T, Wu S. Cerebral white matter changes in young healthy individuals with high trait anxiety: a tract-based spatial statistics study. Front Neurol. 2018;9:704.

19. Kim MJ, Whalen PJ. The structural integrity of an amygdala-prefrontal pathway predicts trait anxiety. J Neurosci. 2009;29:11614-8.

20. Montag C, Reuter M, Weber B, Markett S, Schoene-Bake JC. Individual differences in trait anxiety are associated with white matter tract integrity in the left temporal lobe in healthy males but not females. Neuroscience. 2012;217:77-83.

21. Tromp DPM, Grupe DW, Oathes DJ, McFarlin DR, Hernandez PJ, Kral TRA, et al. Reduced structural connectivity of a major frontolimbic pathway in generalized anxiety disorder. Arch Gen psychiatry. 2012;69:925-34.

22. Phan KL, Orlichenko A, Boyd E, Angstadt M, Coccaro EF, Liberzon I, et al. Preliminary evidence of white matter abnormality in the uncinate fasciculus in generalized social anxiety disorder. Biol Psychiatry. 2009;66:691-4.

23. Hettema JM, Kettenmann B, Ahluwalia V, McCarthy C, Kates WR, Schmitt JE, et al. Pilot multimodal twin imaging study of generalized anxiety disorder. Depress Anxiety. 2012;29:202-9.

24. Neumann A, Muetzel RL, Lahey BB, Bakermans-Kranenburg MJ, van, IJzendoorn $\mathrm{MH}$, Jaddoe $\mathrm{VW}$, et al. White matter microstructure and the general psychopathology factor in children. J Am Acad Child Adolesc Psychiatry. 2020;59:1285-96.

25. Tromp DPM, Williams LE, Fox AS, Oler JA, Roseboom PH, Rogers GM, et al. Altered uncinate fasciculus microstructure in childhood anxiety disorders in boys but not girls. Am J Psychiatry. 2019;176:208-16.

26. Hung Y, Uchida M, Gaillard SL, Woodworth H, Kelberman C, Capella J, et al. Cingulum-callosal white-matter microstructure associated with emotional dysregulation in children: a diffusion tensor imaging study. Neuroimage Clin. 2020;27:102266.

27. Albaugh MD, Ducharme S, Karama S, Watts R, Lewis JD, Orr C, et al. Anxious/ depressed symptoms are related to microstructural maturation of white matter in typically developing youths. Dev Psychopathol. 2016;29:751-8.

28. Jalbrzikowski M, Larsen B, Hallquist MN, Foran W, Calabro F, Luna B. Development of white matter microstructure and intrinsic functional connectivity between the amygdala and ventromedial prefrontal cortex_ associations with anxiety and depression. Biol Psychiatry. 2017;82:511-21.

29. Liao M, Yang F, Zhang Y, He Z, Su L, Li L. White matter abnormalities in adolescents with generalized anxiety disorder: a diffusion tensor imaging study. Bmc Psychiatry. 2014;14:41.

30. Eden AS, Schreiber J, Anwander A, Keuper K, Laeger I, Zwanzger P, et al. Emotion regulation and trait anxiety are predicted by the microstructure of fibers between amygdala and prefrontal cortex. J Neurosci. 2015;35:6020-7.

31. Comte M, Cancel A, Coull JT, Schön D, Reynaud E, Boukezzi S, et al. Effect of trait anxiety on prefrontal control mechanisms during emotional conflict. Hum Brain Mapp. 2015;36:2207-14.

32. Fonzo GA, Ramsawh HJ, Flagan TM, Sullivan SG, Simmons AN, Paulus MP, et al Cognitive-behavioral therapy for generalized anxiety disorder is associated with attenuation of limbic activation to threat-related facial emotions. J Affect Disord. 2014;169:76-85.

33. Williams LE, Oler JA, Fox AS, McFarlin DR, Rogers GM, Jesson MAL, et al. Fear of the unknown: uncertain anticipation reveals amygdala alterations in childhood anxiety disorders. Neuropsychopharmacol: Off Publ Am Coll Neuropsychopharmacol. 2015;40:1428-35.

34. Etkin A, Prater KE, Hoeft F, Menon V, Schatzberg AF. Failure of anterior cingulate activation and connectivity with the amygdala during implicit regulation of emotional processing in generalized anxiety disorder. Am J Psychiat. 2010;167:545-54.

35. Hahn A, Stein P, Windischberger C, Weissenbacher A, Spindelegger C, Moser E, et al. Reduced resting-state functional connectivity between amygdala and orbitofrontal cortex in social anxiety disorder. Neurolmage. 2011;56:881-9.

36. Birn RM, Shackman AJ, Oler JA, Williams LE, McFarlin DR, Rogers GM, et al. Evolutionarily conserved prefrontal-amygdalar dysfunction in early-life anxiety. Mol Psychiatry. 2014;19:915-22.

37. Heide RJVD, Skipper LM, Klobusicky E, Olson IR. Dissecting the uncinate fasciculus: disorders, controversies and a hypothesis. Brain. 2013;136:1692-707.

38. Leng $B$, Han $S$, Bao $Y$, Zhang $H$, Wang $Y, W u ~ Y$, et al. The uncinate fasciculus as observed using diffusion spectrum imaging in the human brain. Neuroradiology. 2016;58:595-606.

39. Tromp DPM, Fox AS, Oler JA, Alexander AL, Kalin NH. The relationship between the uncinate fasciculus and anxious temperament is evolutionarily conserved and sexually dimorphic. Biol Psychiat. 2019;86:890-8.

40. Birmaher B, Ehmann M, Axelson DA, Goldstein BI, Monk K, Kalas C, et al. Schedule for affective disorders and schizophrenia for school-age children (K-SADS-PL) for the assessment of preschool children-a preliminary psychometric study. J Psychiatr Res. 2008;43:680-6. 
41. Busner J, Targum SD. The clinical global impressions scale: applying a research tool in clinical practice. Psychiatry Edgmont Pa Townsh. 2007;4:28-37.

42. Birmaher B, Brent DA, Chiappetta L, Bridge J, Monga S, Baugher M. Psychometric properties of the screen for child anxiety related emotional disorders (SCARED): a replication study. J Am Acad Child Adolesc Psychiatry. 1999;38:1230-6.

43. Saylor CF, Finch AJ, Spirito A, Bennett B. The Children's Depression Inventory: A systematic evaluation of psychometric properties. J Consult Clin Psych. 1984;52:955-67.

44. Williamson DE, Birmaher B, Ryan ND, Shiffrin TP, Lusky JA, Protopapa J, et al. The Stressful Life Events Schedule for children and adolescents: development and validation. Psychiat Res. 2003;119:225-41.

45. Conners CK, Sitarenios G, Parker JDA, Epstein JN. The Revised Conners' Parent Rating Scale (CPRS-R): factor structure, reliability, and criterion validity. J Abnorm Child Psych. 1998;26:257-68.

46. Petersen AC, Crockett L, Richards M, Boxer A. A self-report measure of pubertal status: reliability, validity, and initial norms. J Youth Adolescence. 1988;17:117-33.

47. Harris PA, Taylor R, Thielke R, Payne J, Gonzalez N, Conde JG. Research electronic data capture (REDCap) - a metadata-driven methodology and workflow process for providing translational research informatics support. J Biomed Inf. 2009;42:377-81.

48. Harris PA, Taylor R, Minor BL, Elliott V, Fernandez M, O'Neal L, et al. The REDCap Consortium: Building an International Community of Software Platform Partners. J Biomed Inf. 2019;95:103208.

49. TrackVis [Internet]. [cited 2021 Jul 15]. Available from: http://trackvis.org/.

50. Lai C-H, Wu Y-T. Fronto-occipital fasciculus, corpus callosum and superior longitudinal fasciculus tract alterations of first-episode, medication-naïve and lateonset panic disorder patients. J Affect Disord. 2013;146:378-82.

51. Hyett MP, Perry A, Breakspear M, Wen W, Parker GB. White matter alterations in the internal capsule and psychomotor impairment in melancholic depression. Plos ONE. 2018;13:e0195672.

52. Winkler AM, Ridgway GR, Webster MA, Smith SM, Nichols TE. Permutation inference for the general linear model. Neuroimage. 2014;92:381-97.

53. Chen G, Saad ZS, Britton JC, Pine DS, Cox RW. Linear mixed-effects modeling approach to FMRI group analysis. Neuroimage. 2013;73:176-90.

54. Lagattuta KH, Sayfan L, Bamford C. Do you know how I feel? Parents underestimate worry and overestimate optimism compared to child self-report. J Exp Child Psychol. 2012;113:211-32.

55. Cosi S, Canals J, Hernández-Martinez C, Vigil-Colet A. Parent-child agreement in SCARED and its relationship to anxiety symptoms. J Anxiety Disord. 2010;24:129-33.

56. Davis-Kean PE, Huesmann LR, Jager J, Collins WA, Bates JE, Lansford JE. Changes in the relation of self-efficacy beliefs and behaviors across development. Child Dev. 2008;79:1257-69.

57. Keysers C, Gazzola V, Wagenmakers E-J. Using Bayes factor hypothesis testing in neuroscience to establish evidence of absence. Nat Neurosci. 2020;23:788-99.

58. Nichols TE, Holmes AP. Nonparametric permutation tests for functional neuroimaging: a primer with examples. Hum Brain Mapp. 2002;15:1-25.

59. Smith SM, Nichols TE. Threshold-free cluster enhancement: addressing problems of smoothing, threshold dependence and localisation in cluster inference. Neurolmage. 2009;44:83-98.

60. Brauer M, Curtin JJ. Linear mixed-effects models and the analysis of nonindependent data: a unified framework to analyze categorical and continuous independent variables that vary within-subjects and/or within-items. Psychol Methods. 2018;23:389-411.

61. Muetzel RL, Blanken LME, Ende J, van der, Marroun HE, Shaw P, Sudre G, et al. Tracking brain development and dimensional psychiatric symptoms in children: a longitudinal population-based neuroimaging study. Am J Psychiatry. 2018;175:54-62.

62. Muetzel RL, Mous SE, Ende J, van der, Blanken LME, Lugt A, van der, Jaddoe VWV, et al. White matter integrity and cognitive performance in school-age children: a population-based neuroimaging study. Neuroimage. 2015;119:119-28.

63. Howell BR, McCormack KM, Grand AP, Sawyer NT, Zhang X, Maestripieri D, et al. Brain white matter microstructure alterations in adolescent rhesus monkeys exposed to early life stress: associations with high cortisol during infancy. Biol Mood Anxiety Disord. 2013;3:21.

64. Jensen SKG, Pangelinan $M$, Björnholm L, Klasnja A, Leemans A, Drakesmith $M$, et al. Associations between prenatal, childhood, and adolescent stress and variations in white-matter properties in young men. Neuroimage. 2018;182:389-97.

65. Liu J, Dietz K, DeLoyht JM, Pedre X, Kelkar D, Kaur J, et al. Impaired adult myelination in the prefrontal cortex of socially isolated mice. Nat Neurosci. 2012;15:1621-3.

66. Teissier A, Magueresse CL, Olusakin J, Costa BLSA da, Stasi AMD, Bacci A, et al. Earlylife stress impairs postnatal oligodendrogenesis and adult emotional behaviour through activity-dependent mechanisms. Mol Psychiatr. 2019;25:1159-74.
67. Huber E, Donnelly PM, Rokem A, Yeatman JD. Rapid and widespread white matter plasticity during an intensive reading intervention. Nat Commun. 2018;9:2260.

68. Steele CJ, Bailey JA, Zatorre RJ, Penhune VB. Early musical training and whitematter plasticity in the corpus callosum: evidence for a sensitive period. J Neurosci. 2013;33:1282-90.

69. Scholz J, Klein MC, Behrens TEJ, Johansen-Berg $\mathrm{H}$. Training induces changes in white-matter architecture. Nat Neurosci. 2009;12:1370-1.

70. Mackey AP, Whitaker KJ, Bunge SA. Experience-dependent plasticity in white matter microstructure: reasoning training alters structural connectivity. Front Neuroanat. 2012;6:32.

71. Liu J, Dupree JL, Gacias M, Frawley R, Sikder T, Naik P, et al. Clemastine enhances myelination in the prefrontal cortex and rescues behavioral changes in socially isolated mice. J Neurosci: Off J Soc Neurosci. 2016;36:957-62.

72. Magalhães R, Bourgin J, Boumezbeur F, Marques P, Bottlaender M, Poupon C, et al. White matter changes in microstructure associated with a maladaptive response to stress in rats. Transl Psychiat. 2017;7:e1009-09.

73. Li Z, He Y, Fan S, Sun B. Clemastine rescues behavioral changes and enhances remyelination in the cuprizone mouse model of demyelination. Neurosci Bull. 2015;31:617-25.

74. Abiraman K, Pol SU, O'Bara MA, Chen G-D, Khaku ZM, Wang J, et al. Antimuscarinic adjunct therapy accelerates functional human oligodendrocyte repair. J Neurosci: Off J Soc Neurosci. 2015;35:3676-88.

75. Welliver RR, Polanco JJ, Seidman RA, Sinha AK, O'Bara MA, Khaku ZM, et al. Muscarinic receptor $M 3 R$ signaling prevents efficient remyelination by human and mouse oligodendrocyte progenitor cells. J Neurosci: Off J Soc Neurosci. 2018;38:6921-32.

76. Giorgio A, Watkins KE, Douaud G, James AC, James S, Stefano ND, et al. Changes in white matter microstructure during adolescence. Neuroimage. 2008;39:52-61.

77. Reynolds JE, Grohs MN, Dewey D, Lebel C. Global and regional white matter development in early childhood. Neuroimage. 2019;196:49-58.

\section{ACKNOWLEDGEMENTS}

This research was supported by grant R01MH107563 (NHK) from the National Institute of Mental Health; projects ZIAMH002781 and ZIAMH002782 (DSP) from the Intramural Research Program of the National Institute of Mental Health; and training grants T32GM008692 and T32MH018931 (NA) from the National Institute of General Medical Sciences and National Institute of Mental Health. The project described was supported by the Clinical and Translational Science Award (CTSA) program, through the National Institutes of Health (NIH) National Center for Advancing Translational Sciences (NCATS), grant UL1TR002373. The content is solely the responsibility of the authors and does not necessarily represent the official views of the $\mathrm{NIH}$. The funding sources had no role in the design or conduct of the study; collection, management, analysis, or interpretation of the data; preparation, review, or approval of the manuscript; or decision to submit the manuscript for publication. We thank the participants and families, as well as the staff of the HealthEmotions Research Institute at the Wisconsin Psychiatric Institute and Clinics.

\section{COMPETING INTERESTS}

NHK reported receiving grants from the National Institute of Mental Health; consulting to CME Outfitters, the Pritzker Neuropsychiatric Disorders Research Consortium, the Skyland Trail Advisory Board, the Early Life Adversity Research External Scientific Advisory Board at the University of Texas at Austin, and Corcept Therapeutics Incorporated; and serving as editor-in-chief of The American Journal of Psychiatry during the conduct of the study. The other authors report no potential conflicts of interest.

\section{ADDITIONAL INFORMATION}

Supplementary information The online version contains supplementary material available at https://doi.org/10.1038/s41398-022-01827-y.

Correspondence and requests for materials should be addressed to Nakul Aggarwal.

Reprints and permission information is available at http://www.nature.com/ reprints

Publisher's note Springer Nature remains neutral with regard to jurisdictional claims in published maps and institutional affiliations. 
Open Access This article is licensed under a Creative Commons At c) Atribution 4.0 International License, which permits use, sharing,
adaptation, distribution and reproduction in any medium or format, as long as you give appropriate credit to the original author(s) and the source, provide a link to the Creative Commons license, and indicate if changes were made. The images or other third party material in this article are included in the article's Creative Commons license, unless indicated otherwise in a credit line to the material. If material is not included in the article's Creative Commons license and your intended use is not permitted by statutory regulation or exceeds the permitted use, you will need to obtain permission directly from the copyright holder. To view a copy of this license, visit http://creativecommons. org/licenses/by/4.0/.

(c) The Author(s) 2022 